\title{
Edge-dependent selection rules in magic triangular graphene flakes
}

\author{
J. Akola, H.P. Heiskanen, and M. Manninen \\ NanoScience Center, Department of Physics, P.O. Box 35 FIN-40014 University of Jyväskylä, Finland
}

(Dated: October 30, 2018)

\begin{abstract}
The electronic shell and supershell structure of triangular graphene quantum dots has been studied using density functional and tight-binding methods. The density functional calculations demonstrate that the electronic structure close to the Fermi energy is correctly described with a simple tightbinding model where only the $p_{z}$ orbitals perpendicular to the graphene layer are included. The results show that (i) both at the bottom and at the top of the $p_{z}$ band a supershell structure similar to that of free electrons confined in a triangular cavity is seen, (ii) close to the Fermi level the shell structure is that of free massless particles, (iii) triangles with armchair edges show an additional sequence of levels ('ghost states') absent for triangles with zigzag edges while the latter exhibit edge states, and (iv) the observed shell structure is rather insensitive to the edge roughness.

PACS numbers: 73.21.La, 81.05.Uw, 61.48.De
\end{abstract}

Recent experimental success in manufacturing single layer graphene flakes on various surfaces [1, 2, 3, 4, has made graphene a new playground for theoretical and computational physics, [5, 6, 7, 8, 9] and more and more experimental results are emerging. [10, 11, 12, Most of the recent interest has been focused in the effects caused by the peculiar band structure of graphite near the Fermi level $\left(\epsilon_{\mathrm{F}}\right)$ : Electrons and holes behave as massless particles (Dirac fermions) due to the linear dispersion relation although their velocity is very small. [13.

The triangular shape of two-dimensional clusters is particularly interesting because, in the case of free electrons, it supports perhaps the most persistent and regular supershell structure of all systems. 14] Furthermore, the triangular shape is preferred in two-dimensional metallic systems [15, 16] and in plasma clusters. [17] For tetravalent elements, triangular clusters have been observed in silicon. [18] It is reasonable to expect that such shapes can be observed also for carbon, and this is supported further by the fact that equilateral triangles of graphene can be cut with the two most stable edge structures, the zigzag edge and the armchair edge.

In this letter, we wish to point out that finite graphene flakes (or quantum dots) have an intriguing energy spectrum close to the Fermi level. We have performed electronic structure calculations for triangular graphene flakes using the density functional theory (DFT) for all the valence electrons, and a tight-binding (TB) approach that considers only the carbon $p_{z}$ electrons (Hückel model). Our results show that already in small triangular flakes $(N=300, L=5 \mathrm{~nm})$ the electronic levels close to $\epsilon_{\mathrm{F}}$ can be understood as those of free massless electrons confined in a triangular cavity. Especially, we demonstrate that the edge structure has a selective role in the electronic shell structure: The zigzag edge prohibits a whole sequence of localized states inside the cluster although it supports edge states. This leads to well-defined edgedependent selection rules that are based on an analytical model. Recently, Yamamoto et al. addressed the pres- ence (absence) of edge states at $\epsilon_{\mathrm{F}}$ in zigzag (armchair) triangles of graphene, and the effect on the optical absorption, [19] but the simple principles of the underlying energy spectrum have remained unexplained.
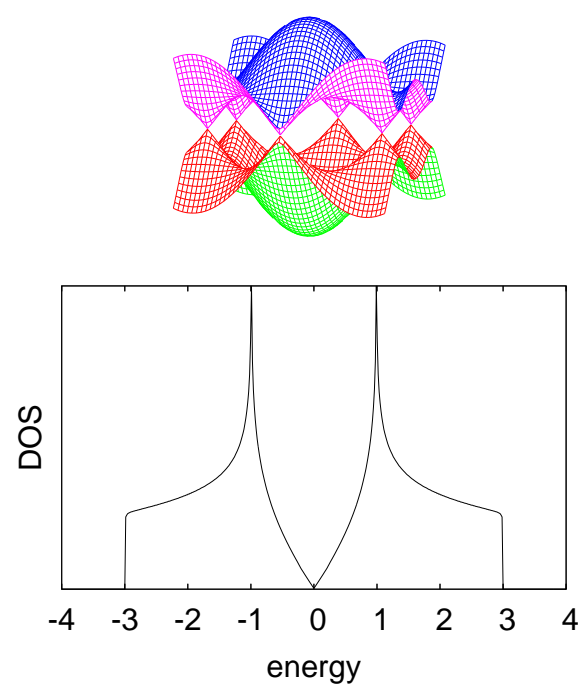

FIG. 1: Cross-over of the valence and conduction bands at the Fermi energy (top), and the density of states (bottom, $p_{z}$ electrons) of an infinite graphene sheet.

It is well-known that the atomic $p_{z}$ electrons perpendicular to the graphene plane are responsible for the captivating band structure shown in Fig. 1 with the valence the conduction bands meeting at the corners of the hexagonal Brillouin zone. [20, 21] The Fermi surface consists of a discrete set of these points of high- $k$ value, and the resulting density of states (DOS) has a zero weight at $\epsilon_{\mathrm{F}}$. The cross-over regions have locally hourglass-like shapes, which results in the linear and isotropic electron dispersion relation in the conduction band $\left(\epsilon>\epsilon_{F}=0\right)$ but only in a small energy interval. Since the atomic $p_{z}$ 
electrons are perpendicular to the graphene plane their interaction with the neighboring atoms does not have any directional dependence and, consequently, they can be described as $s$-type electrons in the TB model. Neglecting also the differential overlap between atomic sites the system can be described with the traditional Hückel model

$$
H_{i j}=\left\{\begin{aligned}
-t, & \text { if } i, j \text { nearest neighbours } \\
0, & \text { otherwise }
\end{aligned}\right.
$$

where the hopping parameter $t$ (resonance integral) determines the width of the bands and the on-site energy is chosen to be $\epsilon_{F}=0$. We choose to present our results in units $t=1$. The resulting TB bands (Fig. 1) reaches from -3 to +3 (in real graphene our unit $t$ corresponds to about $2.6 \mathrm{eV})$.

A conceptual cutting of a finite graphene flake breaks covalent bonds yielding edges with dangling bonds. We consider the dangling bonds to be passivated, say with hydrogen. Since the covalent bonding with hydrogen involves $s p^{2}$ hybridized orbitals, the passivation is expected to have only a small effect on the perpendicular $p_{z}$ electron states. Therefore, we neglect this effect in our TB model and follow Areshkin et al. 21] and treat the edge atoms in the same footing as bulk atoms. Moreover, we will completely neglect the interaction of graphene with the possible substrate and treat the graphene flake as an isolated two-dimensional cluster or quantum dot. As we shall see, the results of the simple TB model agree well with those of the full DFT calculation.

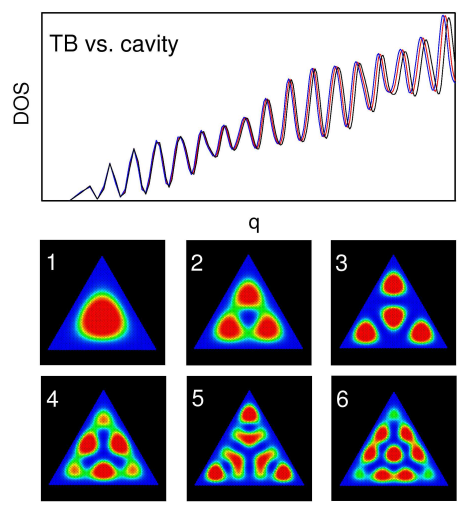

FIG. 2: Upper panel: DOS at the bottom of the TB band shown as a function of $q=\sqrt{\epsilon+3 t}$. Blue: zigzag triangle with 10000 atoms; red: armchair triangle with 9918 atoms; and black: result for free electrons in a triangular cavity. Lower panel: Electron densities of the six lowest energy levels.

It has been shown that at the bottom of the valence band the TB model gives exactly the free electron states for a triangular lattice,22. and the same is true also for the hexagonal graphene. Consequently, at the bottom (and at the top) the energy levels are expected to show the same shell structure as free electrons in a triangular cavity, determined by the equation [23, 24]

$$
\epsilon_{n, m}=\epsilon_{0}\left(n^{2}+m^{2}-n m\right),
$$

where $\epsilon_{0}=8 \pi^{2} \hbar^{2} / 3 m_{e} L^{2}, L$ being the length of the triangle side. The quantum numbers must satisfy $m \geq 1$ and $n \geq 2 m$. Determination of the electron effective mass in the graphene lattice gives for the TB model $\epsilon_{0}=4 \pi^{2} t / 9 N$, where $N$ is the number of atoms in the triangle ( $L=3 d \sqrt{N} / 2$ for a large triangle, $d$ is the nearestneighbor distance).

The shell structure manifests itself as a regular variation of the density of the states (DOS) which can be determined by Gaussian convolution of the discrete levels. Figure 2 shows DOS close to the bottom of the valence band obtained from the above equation and compared to the TB model for two graphene triangles, one with 10000 atoms (zigzag edge) and the other with 9918 atoms (armchair edge). The profiles are clearly similar and exhibit the beating pattern of the supershell structure 17. Note that DOS is plotted as a function of $\sqrt{\epsilon+3 t}$ making the shells equidistant. Fig. 2 shows also the electron densities corresponding to the six lowest energy levels (for degenerate states we show the sum of the density). The density patterns are identical to those of free electrons confined in a triangle [16] or wave modes in triangular resonators [26].

The Fermi level of graphene consists of two equivalent points at the border of the Brillouin zone (see Fig. 1) where the conduction and valence bands open as circular cones resulting a linear dispersion relation for electrons $\epsilon(\mathbf{k})=C \hbar k$, where $C$ is the velocity. Thus, it is to be expected that the electron dynamics is not determined by the Schrödinger equation but by the wave equation of massless particles (or the Dirac equation). For free particles confined in a triangle the energy levels are still determined by Eq. (1) but now it results in the square of the energy, i.e

$$
\epsilon_{n, m}=\epsilon_{1} \sqrt{n^{2}+m^{2}-n m},
$$

where $\epsilon_{1}=2 \pi t / \sqrt{3 N}$. It is interesting to note that these energy levels were actually computed for the wave equation much earlier than for the Schrödinger equation. 23 .

Figure 3 shows TB-DOS above the Fermi energy for two large triangles ( $\sim 10000$ atoms) with zigzag and armchair edges and compares them with the levels of free massless electrons [Eq. [2]]. The results are the following: (i) Each energy level has an additional degeneracy of two due to the two equivalent points at $\epsilon_{F}$. (ii) The zigzag triangle shows the levels of Eq. (2) with index values $m \geq 1$ and $n \geq 2 m$ while the armchair edge shows 

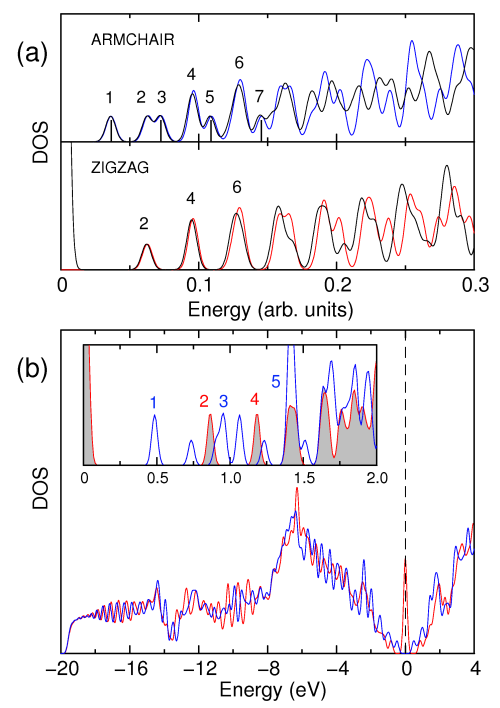

FIG. 3: Upper panel: TB-DOS at the Fermi level displayed as a function of energy (red and blue curves) compared to the density of levels of Eq. (2) (black curves). The zigzag triangle has 10000 atoms and the armchair triangle 9918 atoms. Lower panel: DOS of the full DFT calculation for the triangular $\mathrm{C}_{321} \mathrm{H}_{51}$ (zigzag, red) and $\mathrm{C}_{330} \mathrm{H}_{60}$ (armchair, blue) flakes. The inset shows the levels above the Fermi surface where the zigzag spectrum is shaded.

all the levels where $n \geq m \geq 1$. (iii) The states are much less dense than at the bottom of the band, and Eq. 22 describes only the lowest states accurately. (iv) Due to the sparseness of the states, no supershell oscillations are visible for the massless particles (although the supershell structure of ordinary electrons is clearly seen in Fig. 2). (v) The zigzag edge supports particularly visible edge states [27, 28, that appear at $\epsilon_{F}$ as a prominent peak. The number of these states equals the number of the outermost edge atoms in zigzag triangles, which is $N_{\mathrm{ss}}=\sqrt{N}$.

In order to compare our results with a more realistic calculation, we have performed DFT calculations for triangular $\mathrm{C}_{321} \mathrm{H}_{51}$ (zigzag) and $\mathrm{C}_{330} \mathrm{H}_{60}$ (armchair) flakes with the CPMD program. 29] The DFT calculations use a plane wave basis set $\left(E_{c u t}=50 \mathrm{Ry}\right)$, pseudopotentials, [30] and a generalized gradient-corrected PBE approximation for the exchange-correlation energy. 31] The resulting DFT-DOS of all valence electrons is plotted in Fig. 3(b) for both systems, and they show overall features characteristic for graphite. The zigzag edge states at $\epsilon_{F}$ are visible, and the closest conduction states obey the simple analytical model of Eq. 22. The even-numbered peaks are split for the armchair triangle, which is a result reproduced by TB (the splitting reduces with increasing system size).
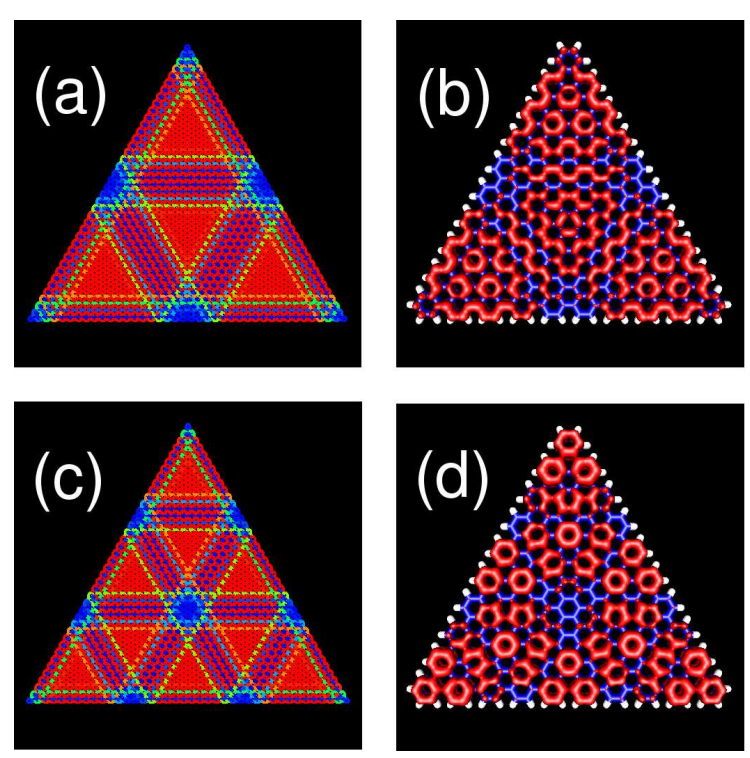

FIG. 4: Electron density of the 3rd [(a) and (b)] and 5th $[(c)$ and (d)] energy levels above the Fermi energy in armchair triangles ('ghost states', labeled in Fig. 3. each has a degeneracy two). (a) and (c) are computed for a large TB triangle of $4920 \mathrm{C}$ atoms while (b) and (d) are DFT results for a $\mathrm{C}_{330} \mathrm{H}_{60}$ molecule.

The lowest conduction states that are labeled in Fig. 3 show fascinating details, and the electron densities of two such states are visualized in Figure 4. For comparison, we show the same states/orbitals calculated for a large triangle with the TB model (4920 C atoms) and for a small triangle calculated with the DFT method (330 C atoms). The internal structure (symmetry) of the states is clearly similar, and therefore, it is independent of the triangle size and the model used. The states close to the Fermi level appear very different from those at the bottom of the band (Fig. 2). They are not simple densities of massless particles confined in a triangle since the density profile does not decay to zero at the edges. The corresponding electron levels are close to the Brillouin zone boundary, having large $k$-values, and the wave functions have pronounced oscillations with wave lengths that are related to the unit cell size. These oscillations guarantee that the wave function will be formally zero at the edges, but the corresponding pseudowave function of the massless particle does not necessarily show the same behavior. An interesting feature in Fig. 4 is that the states have simple geometric structure of triangular symmetry. The size (number) of the triangles decreases (increases) with increasing energy, i.e. the pattern repeats itself. These 'ghost states' are completely absent for the zigzag triangles, and they correspond to quantum numbers of Eq. (2) not allowed for free electrons in a triangular box [i.e. $2 m \geq n \geq m \geq 1$ in Eq. [2]]. 

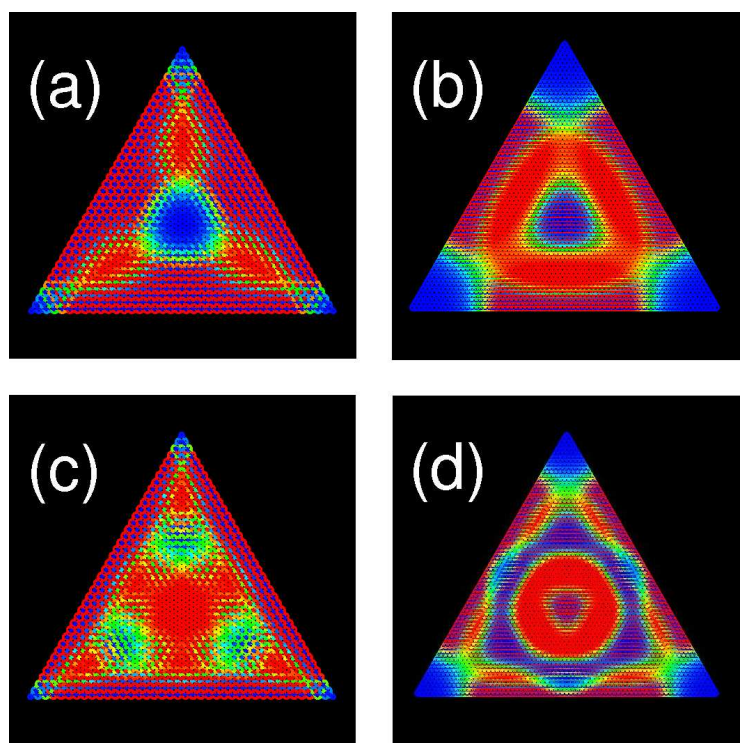

FIG. 5: Electron density (TB model) of the 2nd [(a) and (b)] and 4 th $[(\mathrm{c})$ and (d)] energy levels above the Fermi energy (labeled in Fig. 3) for armchair and zigzag triangles of 4920 and $5181 \mathrm{C}$ atoms, respectively.

Figure 5 shows the electron densities corresponding to the 'normal' low energy states that obey the standard selection rules $(m \geq 1$ and $n \geq 2 m)$. Again, the electron density does not necessarily vanish at the edges of the triangle. The corresponding states for the armchair and zigzag triangles display obvious differences despite the fact that they involve the same set of quantum numbers (and energy).

Finally, we want to note that a small roughness of the edge does not remove the peculiar states shown in Fig. 4 or change the shell structure close to the Fermi level. These 'ghost states' form a triangular network, and it would be interesting to study if they can exist also in the graphene flakes with hexagonal, parallelogram, or trapezoidal shapes.

In conclusion, we have computed the electronic structure of triangular graphene flakes and shown that the DOS profile close to $\epsilon_{F}$ is independent of the triangle size, and it can be described with the simple TB model. The zigzag flakes exhibit well-known edge states, and the armchair triangles show an additional set of 'ghost states' (different selection rules) where the corresponding electron density makes a triangular lattice. In large triangles of 5000-10000 C atoms, the energy levels can be described accurately by considering free massless particles confined in a triangular cavity. Presumably, the electronic states near the Fermi surface are not sensitive to the dielectric substrate, and we expect that these fascinating wave functions can be observed with scanning tunneling microscopy.
[1] C. Berger, Z.M. Song, T.B. Li, X.B. Li, A.Y.Ogbazghi, R. Feng, Z.T. Dai, A.N. Marchenkov, E.H. Conrad, P.N. First, W.A. de Heer, J. Phys. Chem. 108, 19912 (2004).

[2] K.S. Novoselov, A.K. Geim, S.V. Morozov, D. Jiang, Y. Zhang, S.V. Dubonos, I.V. Grigorieva, A.A. Firsov, Science 306666 (2004).

[3] K.S. Novoselov, A.K. Geim, S.V. Morozov, D. Jiang, M.I. Katsnelson, I.V. Grigorieva, S.V. Dubonos, A.A. Firsov, Nature 438, 197 (2005).

[4] C. Berger, Z.M. Song, X.B. Li, X.S. Wu, N. Brown, C. Naud, D. Mayo, T.B. Li, J. Hass, A.N. Marchenkov, E.H. Conrad, P.N. First, W.A. de Heer, Science 312, 1191 (2006).

[5] J. Alicea J and M.P.A. Fisher, Phys. Rev. B 74, 75422 (2005).

[6] V.P. Gusynin, and S.G. Sharapov, Phys. Rev. Lett. 95, 146801 (2005).

[7] V.P. Gusynin, S.G. Sharapov, J.P. Carbotte, Phys. Rev. Lett. 96, 256802 (2006).

[8] K. Nomura, A.H. MacDonald, Phys. Rev. Lett. 98, 76602 (2007).

[9] Y.-W. Son, M.L. Cohen, S.G. Louie, Phys. Rev. Lett. 97, 216803 (2007).

[10] K.S. Novoselov, Z. Jiang Z, Y. Zhang, S.V. Morozov SV, H.L. Stormer, U. Zeitler, J.C. Maan, G.S. Boebinger, P. Kim, A.K. Geim, Science 3151379 (2007).

[11] A.K. Geim and K.S. Novoselov, Nature materials 6, 183 (2007).

[12] G. Li and E.A. Andrei, Nature Phys. 3, 623 (2007).

[13] S.Y. Zhou, G.H. Gweon, J. Graf, A.V. Fedorov, C.D. Spataru, R.D. Diehl, Y. Kopelevich, D.-H. Lee, S.G. Louie, A. Lanzara, Nature Phys. 2, 595 (2006).

[14] M. Brack, J. Blaschke, S.C. Greagh, A.G. Magner, P. Meier, and S.M. Reimann, Z. Phys. D 40,276 (1997).

[15] J. Kolehmainen, H. Häkkinen, and M. Manninen, Z. Phys. D 40, 306 (1997).

[16] E. Jenssens, H. Tanaka, S. Neukermans, R.e. Silverans, and P. Lievens, New J. Phys. 5, 46 (2003).

[17] S.M. Reimann, M. Koskinen, J. Helgesson, P. E. Lindelof, and M. Manninen, Phys. Rev. B 58, 8111 (1998).

[18] M.Y. Lai and Y.L. Wang,Phys. Rev. Lett. 81, 164 (1998).

[19] T. Yamamoto, T. Noguchi, and K. Watanabe, Phys. Rev. B 74, 121409 (2006).

[20] S.R. Elliot, The Physics and Chemistry of Solids (John Wiley \& Sons, New York 1998).

[21] D.A. Areshkin, D. Gunlycke, and C.T. White, Nano Lett. 7, 204 (2007).

[22] M. Manninen, J. Mansikka-aho, E. Hammarén, Europhys. Lett. 15, 423 (1991).

[23] F.E. Borghis and C.H. Papas, in Encyclopedia of Physics, ed. by S. Flücke (Springer, Berlin 1957).

[24] H.R. Krishnamurthy, H.S. Mani, and H.C. Verma, J. Phys. A: Math. Gen. 15, 2131 (1982).

[25] S.M. Reimann, M. Koskinen, H. Häkkinen, P.E. Lindelof, and M. Manninen, Phys. Rev. B 56, 12147 (1997).

[26] Y.-Z. Huang, W.-H. Guo, L.-J. Yu, and H.-B. Lei, IEEE J. Quantum Electrodynamics 37, 1259 (2001).

[27] K. Kobayashi, Phys. Rev. B 48, 1757 (1993).

[28] K. Nakada, M. Fujita, G. Dresselhaus, M.S. Dresselhaus, Phys. Rev. B 54, 17954 (1996).

[29] CPMD V3.11 Copyright IBM Corp 1990-2006, Copy- 
right MPI für Festkörperforschung Stuttgart 1997-2001. www.cpmd.org.

[30] N. Troullier and J.L. Martins, Phys. Rev. B 43, 1993 (1991).
[31] J.P. Perdew, K. Burke, and M. Ernzerhof, Phys. Rev. Lett. 77, 3865 (1996). 